\title{
A Nationwide Study of the Prevalence and Initial Management of Atypical Genitalia in the Newborn in Scotland
}

\author{
Martina E. Rodie ${ }^{a, b}$ Salma R. Ali ${ }^{b}$ Arundathi Jayasena ${ }^{b, c}$ Naser R. Alenazi ${ }^{b} d$ \\ Martin McMillan $^{b}$ Kathryn Cox ${ }^{e}$ Sumaiya M. Cassim $^{f}$ Stuart Henderson $^{b, g}$ \\ Ruth McGowan ${ }^{b, h}$ S. Faisal Ahmed ${ }^{b}$ on behalf of the Scottish DSD Network \& \\ the Scottish Paediatric Endocrine Group
}

\begin{abstract}
${ }^{a}$ Neonatal Intensive Care Unit, Royal Hospital for Children, Glasgow, UK; ${ }^{\text {b }}$ Developmental Endocrinology Research Group, University of Glasgow, Glasgow, UK; 'P Paediatric Department, Leeds Teaching Hospital, Leeds, UK;

dPaedatric Diabetes and Endocrinology Unit, Al-Ahmadi Hospital, Kuwait, Kuwait; ' Royal Hospital for Sick Children, NHS Lothian, Edinburgh, UK; fNeonatal Intensive Care Unit, Wishaw General Hospital, Wishaw, UK; 9Paediatric

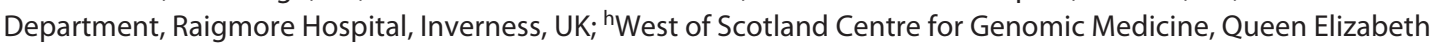
University Hospital, Glasgow, UK
\end{abstract}

\section{Keywords}

Atypical genitalia - Disorder of sex development

\begin{abstract}
Provision of optimum healthcare for infants with atypical genitalia requires a clear understanding of the occurrence of this condition. The objective of this study was to determine the prevalence of atypical genitalia and its initial management. A prospective, electronic survey of clinicians within managed clinical networks in Scotland was undertaken between 2013 and 2019. Notification from clinicians was sought for term neonates requiring specialist input for atypical genitalia. Additional information was also sought from the 4 regional genetics laboratories that provided details for neonates who had an urgent karyotype performed for atypical genitalia or sex determination. In total, the study identified 171 term infants who required some investigation for atypical genitalia in the neonatal period, providing a birth
\end{abstract}

prevalence of 1:1,881 term births. Of the 171 infants, 97 (57\%) had specialist input over the first 3 months of life, providing a birth prevalence of 1:3,318 term births that received specialist input for atypical genitalia. A total of 92 of these 97 cases had complete 3-month follow-up data, 62 (67\%) presented within $24 \mathrm{~h}$ of birth, and age at presentation ranged from birth to 28 days. Age at sex assignment ranged from birth to 14 days, and in 63 cases (68\%), sex assignment occurred at birth. Thus, the birth prevalence of a case of atypical genitalia where sex assignment was reported to be delayed beyond birth was estimated at 1:11,097 births. In 1 case sex was re-assigned at 3 months. Atypical genitalia requiring specialist input within the first month of life are rare in term newborns, and in only a third of these cases, sex assignment is delayed beyond birth. This study provides new clinical benchmarks for comparing and improving the delivery of care in centres that manage these conditions.

(c) 2021 The Author(s)

Published by S. Karger AG, Basel karger@karger.com www.karger.com/sxd

Karger $\stackrel{\text { ' }}{5}$

BOPEN ACCESS
(C) 2021 The Author(s)

Published by S. Karger AG, Basel

This is an Open Access article licensed under the Creative Commons Attribution-NonCommercial-4.0 International License (CC BY-NC) (http://www.karger.com/Services/OpenAccessLicense), applicable to the online version of the article only. Usage and distribution for commercial purposes requires written permission.
Correspondence to:

Martina E. Rodie, martina.rodie@ glasgow.ac.uk 


\section{Introduction}

Although sex assignment usually occurs immediately after birth, in situations where the child is born with atypical genitalia, assignment may be delayed or the child may undergo sex reassignment within a few days of birth. A delay in sex assignment or the event of reassignment is extremely stressful for parents [Sandberg et al., 2012]. Current guidelines highlight the need for expert communication, emotional support, and thorough clinical evaluation during this period of potential uncertainty [Hughes et al., 2006; Ahmed et al., 2016]. To plan health care during this period, there is a need to understand the prevalence of atypical genitalia. Population studies using linked hospital datasets suggest that atypical genitalia may occur in 1:300 births [Ahmed et al., 2004]. On the other hand, complex forms of atypical genitalia have been reported to occur in about 1:1,000 births [Aydin et al., 2019]. Whilst a recent hospital-based study has also reported a similar birth prevalence in the immediate neonatal period [Rodie et al., 2011], another study has reported that atypical genitalia may be much commoner occurring in almost $2 \%$ of births [Ameyaw et al., 2019]. In some cases of atypical genitalia, especially the complex forms, immediate sex assignment may not be possible, and it is recommended that in such cases sex assignment should be delayed [Acherman and Hughes et al., 2017]. Ambiguous genitalia where sex assignment on expert examination is difficult is reported to occur in about 1:5,000 births [Thyen et al., 2006]. However, the extent of variation in the genitalia is a continuum, and it is possible that delayed sex assignment may occur more frequently. Currently, there are no published reports that have systematically studied the extent of delay in sex assignment. A possible marker of delayed sex assignment is the infant with atypical genitalia who requires chromosome analysis, traditionally by karyotype, in the immediate neonatal period. Given that the karyotype is also a recommended first-line investigation in all cases that require multidisciplinary specialist input for a suspected disorder of sex development (DSD) [Ahmed et al., 2016], an assessment of its performance may also allow an understanding of the cases that may be considered to have genitalia that are sufficiently atypical to require expert evaluation. A previous registry-based study suggested that a karyotype may be performed within the first 10 days in about 1:1,300 infants [Rodie et al., 2011]. Thus, the occurrence of atypical genitalia is very variable and depends primarily on the definition of this condition. Clarity of evidence in this field would not only allow improved service delivery through the develop- ment of care pathways and targeted education and training but would also allow the development of benchmarks of clinical practice. We believe that the defining of atypical genitalia by the timing of sex assignment is not only a more objective method of capturing the occurrence of atypical genitalia but it also allows the integration of a measure of quality of care into this statistic.

In Scotland, the clinical care of a range of rare conditions in children and adults is coordinated through managed clinical networks (MCN), such as the multidisciplinary Scottish DSD Network (SDSD) (www.sdsd.scot. nhs.uk) and the Scottish Paediatric Endocrine Group (SPEG) (www.speg.scot.nhs.uk). Members of these 2 networks form the entire group of specialist clinicians who would become involved in any case of an infant with atypical genitalia that requires expert input. The aim of the current study was to utilise these clinical networks to analyze the prevalence and understand the presentation of atypical genitalia in the newborn period, focusing primarily on delayed sex assignment and its management.

\section{Methods}

Monthly emails were sent to all consultant pediatricians, pediatric surgeons, pediatric urologists, and pediatric endocrinologists who were members of SDSD and SPEG between July 2013 and December 2019, asking them to report any cases of atypical genitalia. Case notification was restricted to infants who were $\geq 37$ weeks gestation with any form of atypical genitalia that required specialist pediatric surgical or endocrine input at $<4$ weeks of age. Preterm infants were not included in this study as assessment of their genitalia is often complicated by the effects of prematurity on the genitalia. Positive notifications were followed-up by a questionnaire that enquired about the care received at the centre where the child was born as well as the specialist centre where the child may have been referred or transferred to for specialist care over the first 3 months of life (online suppl. Table 1 and suppl. Fig. 1, 2; see www.karger.com/doi/10.1159/517327 for all online suppl. material). Clinical details were also obtained through this questionnaire. The extent of virilisation of the external genitalia was described as the external masculinisation score (EMS) and as previously reported [Ahmed et al., 2000]. Clinicians who did not respond to the monthly email for 3 consecutive months were removed from the email circulation list. Birth prevalence was calculated by using annual live birth rates in Scotland as the denominator (https://www.nrscotland.gov.uk). Term birth rate was calculated using a figure of $8 \%$ preterm births in Scotland (www. europeristat.com).

In addition, all 4 regional NHS genetics laboratories that cover Scotland were contacted every 6 months from July 2013 to December 2019 to obtain information on all newborns that had an urgent karyotype within the first 4 weeks of life for atypical genitalia or sex assignment using the codes "sex" or "genitalia". The requesting clinician was sent the study questionnaire to complete.
Rodie et al. 


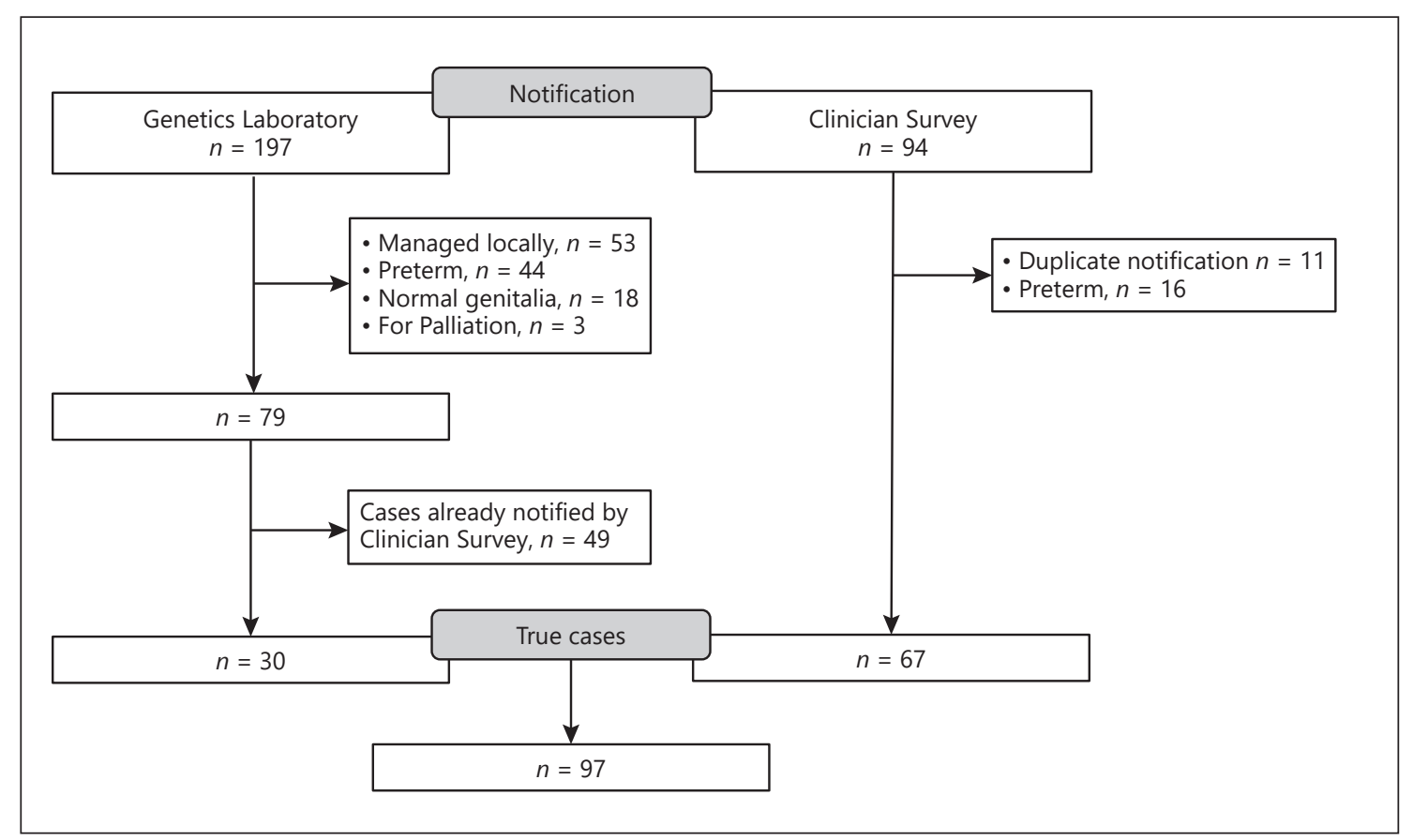

Fig. 1. Total number of cases notified by the survey and identified through the genetics laboratories.

All continuous data are described as medians and ranges. Intergroup comparison of categorical and continuous variables was performed using the $\chi^{2}$ test and the Mann Whitney $U$ test, respectively, and $p<0.05$ was considered to be an appropriate level of significance.

\section{Results}

\section{Monthly Survey}

Between July 2013 and December 2019 the number of monthly responses ranged from 16 to 22 , and the median (range) monthly response rate to the survey was $78 \%(55$, 91). Over this period, 94 infants were reported through the surveys. Of these infants, 67 (71\%) fulfilled the inclusion criteria and of the remaining 27 infants, 16 were excluded because of prematurity, and the remaining 11 were duplicate notifications. An additional 30 cases were identified through the regional genetic laboratories providing a total of 97 cases (Fig. 1). Using the total live term birth rate of 321,811 births in Scotland over the duration of the survey, the birth prevalence of atypical genitalia involving specialist input within the first month of birth in term newborns in Scotland was calculated as 1:3,318. Of these 97 cases, data up to 3 months of age were available in $92(95 \%)$.

\section{Cases Not Fulfilling all Notification Criteria}

There is one group of infants amongst those who did not fulfil all the notification criteria that deserves further attention. Of the 197 infants who had a karyotype performed by the genetics laboratories, 44 were excluded as preterm, 49 had already been notified through the clinician survey, and 30 were identified as true cases which had not been notified. This left a group of 74 infants, of which 18 had normal genitalia, 3 died, and 53 were managed in their local centres without specialist input within the first 4 weeks of life (Fig. 1). The presentation in these 53 infants included bilateral undescended testes in 34 (64\%), hypospadias in 6 (11\%), suspected clitoromegaly in $7(13 \%)$, concerns regarding a prenatal sex chromosome variation in $2(4 \%)$, micropenis in $2(4 \%)$, and nonspecific atypical genitalia in 2 (4\%). Of these 53 cases, 29 (55\%) were referred for a specialist endocrine or surgical opinion after the age of 4 weeks. These included 22 cases of bilateral undescended testes, 6 hypospadias, and 1 sex chromosome variation $(47, \mathrm{XXY})$. When the 74 term infants who had an urgent karyotype performed but did not have specialist input were combined with the 97 cases that did have specialist input (total 171), for calculation of the birth prevalence of atypical genitalia where there is sufficient concern to perform a karyotype within the neonatal period, this resulted in a figure of 1:1,881 births (Fig. 1). 
Fig. 2. Age at sex assignment and external masculinisation score (EMS) in the infant with a XY karyotype or with a karyotype containing a $\mathrm{Y}$ chromosome.

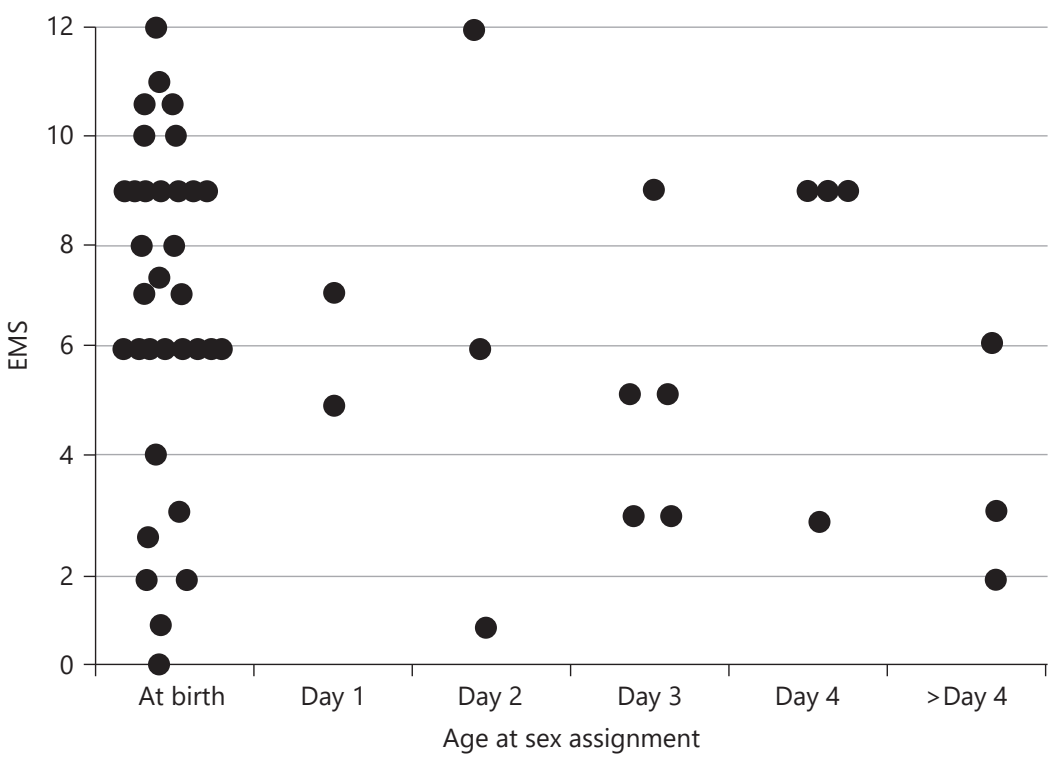

\section{Description of Presentation}

Of the 92 term infants with complete 3-month data, 43 (47\%) presented at birth, $20(22 \%)$ presented within $24 \mathrm{~h}$ of birth, and the remainder presented after $24 \mathrm{~h}$. Of these 92 infants, 64 (70\%) and 20 (22\%) were 46,XY and 46,XX, respectively, whilst the remaining $8(8 \%)$ had a range of sex chromosome variations with a Y chromosome complement. Of the 92 infants, 40 (43\%) had required a period of intensive care, high dependency, or special care. For XY cases, diagnostic categories included non-specific XY DSD $(n=55)$, complete androgen insensitivity syndrome (CAIS) $(n=3)$, bladder exstrophy $(n=2)$, partial gonadal dysgenesis $(n=2)$, congenital adrenal hyperplasia $(\mathrm{CAH})(n=1)$, and persistent Müllerian duct syndrome $(n=1)$. For XX cases, diagnoses included congenital adrenal hyperplasia $(n=8)$, non-specific clitoromegaly $(n=5)$, atypical genitalia $(n=4)$, cloacal anomalies $(n=2)$, and disorder of Müllerian development $(n=1)$. This last case was detected as the infant was admitted for neonatal care for an imperforate anus.

\section{Sex Assignment}

Of the 92 term infants with complete 3-month data, $66(72 \%)$ were initially assigned a male sex and $26(28 \%)$ were assigned a female sex. Of the 72 infants who were $46, \mathrm{XY}$ or had a Y chromosomal complement, 66 (92\%) were assigned a male sex and the remainder a female sex. Of the 2046 ,XX infants, 19 (95\%) were assigned a female sex, and 1 infant was assigned male but re-assigned to the female sex when she was diagnosed with congenital adrenal hyperplasia at 28 days of life. Thus, 6 out of 26 (23\%) assigned a female sex were $46, \mathrm{XY}$ or had a Y chromosomal complement. The age at sex assignment ranged from birth to 14 days. Of the 92 infants, 63 (68\%) had sex assignment at birth, 4 (4\%) by day 1 but not at birth, 24 (26\%) by day 7 , and 1 (1\%) by day 14 (Fig. 2). Thus the birth prevalence of a case of atypical genitalia where the sex assignment was reported to be delayed beyond birth was estimated at 1:11,097 term infants. The 13 cases who were $46, \mathrm{XY}$ or had a Y chromosomal complement, and in whom sex was assigned on day 3 or later, included non-specific XY DSD $(n=10)$ and partial gonadal dysgenesis $(n=3)$. The median (range) EMS in this group who were assigned sex on day 3 or later was $5(2,9)$ compared to $8(0,12)$ for those assigned sex at an earlier age $(p=0.047)$.

\section{Involvement of Specialist Multidisciplinary Team}

Of the 92 cases, 86 (93\%) of the families met a neonatologist or general paediatrician within the first 3 months, $68(74 \%)$ a paediatric endocrinologist, $59(64 \%)$ a paediatric surgeon or urologist, 21 (23\%) a clinical geneticist, $20(22 \%)$ an endocrine nurse specialist, and $12(13 \%)$ a clinical psychologist (Fig. 3). In the 72 infants who had an $\mathrm{XY}$ karyotype or a Y chromosome complement, the median (range) number of health professionals encountered 
Fig. 3. Percentage of healthcare professionals involved in the care of XY DSD infants by EMS category. EMS, external masculinisation score.

Table 1. Investigations performed within the first 3 months of life in the 72 infants with a Y chromosome complement with atypical genitalia at birth.

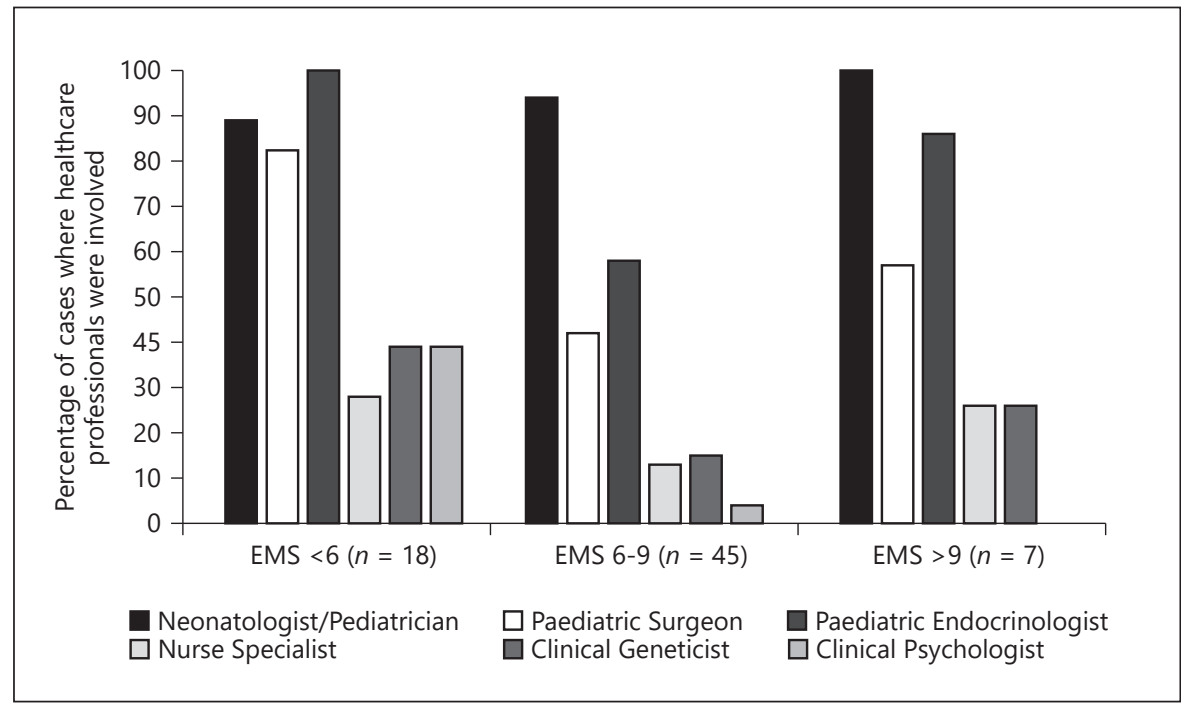

\begin{tabular}{llcl}
\hline & $\begin{array}{l}\text { Number of } \\
\text { infants }(\%)\end{array}$ & $\begin{array}{l}\text { Median age at } \\
\text { test, days (range) }\end{array}$ & $\begin{array}{l}\text { Median EMS } \\
\text { (range) }\end{array}$ \\
\hline Urea and electrolytes & $57(79)$ & $2(1,24)$ & $8(0,12)$ \\
Ultrasound scan of pelvis & $52(72)$ & $3(1,58)$ & $7(0,12)$ \\
Testosterone & $51(71)$ & $3(1,86)$ & $7(0,12)$ \\
Anti-Müllerian hormone & $43(60)$ & $3(1,30)$ & $7(0,10)$ \\
Androstenedione & $39(54)$ & $3(1,86)$ & $8(1,12)$ \\
Glucose & $28(39)$ & $2(1,7)$ & $7(1,12)$ \\
Cortisol & $28(39)$ & $3(1,11)$ & $6(1,9)$ \\
Urinary steroid analysis & $24(33)$ & $5(1,46)$ & $6(1,12)$ \\
Luteinizing hormone & $20(28)$ & $3(1,77)$ & $6(1,9)$ \\
Follicle stimulating hormone & $19(26)$ & $3(1,77)$ & $6(1,9)$ \\
Dihydrotestosterone & $11(15)$ & $5(3,86)$ & $6(2,9)$ \\
ACTH level & $10(14)$ & $6(3,27)$ & $9(4,9)$ \\
HCG stimulation & $8(11)$ & $33(1,89)$ & $9(0,10)$ \\
\end{tabular}

ACTH, adrenocorticotrophic hormone; HCG, human chorionic gonadotrophin by those cases with an EMS $<6$, EMS 6-9, and EMS $>9$ was $4(2,6), 2(2,5)$, and $3(2,5)$, respectively (Fig. 3$)$. Of the 69 infants where psychology information was available, the median (range) EMS of the infants whose parents met a psychologist $(n=12)$ and did not meet a psychologist $(n=57)$ was $3(0,9)$ and $8(0,12)$, respectively $(p=0.01)$. Of the 18 infants with an XY karyotype or who had a Y chromosome complement who had delayed sex assignment after birth, the parents of 4 infants (22\%) met a psychologist. Of the 18 infants with an XY karyotype or who had a Y chromosome complement and an EMS $<6,7$ (39\%) had involvement with a psychologist and of these, 4 (57\%) had delayed sex assignment.

\section{Investigations}

Other than the karyotype, the more common investigations performed in cases of 46,XX DSD were serum urea and electrolytes, pelvic ultrasound scan, and serum glucose levels within the first 3 months of life. In the 72 infants with an XY karyotype or a Y chromosome complement, the more common investigations, other than the karyotype, within the first 3 months were serum urea and electrolytes, serum testosterone, and pelvic ultrasound scan (Table 1). Apart from the hCG stimulation test and serum dihydrotestosterone, all investigations, including analysis of urinary steroid excretion, were usually performed within the first week of life. 


\section{Discussion}

Over a period exceeding 7 years, the birth prevalence of atypical genitalia requiring specialist input by a member of a managed clinical network within the first month of life in term newborns in Scotland was calculated as 1:3,318. This is more common than previously reported and probably reflects the differences in definition [Thyen et al., 2006]. The current study had not included preterm infants as assessment of their genitalia is often complicated by the effects of prematurity on the genitalia. Cross referencing of the reported cases with the regional cytogenetic laboratories proved to be an additional strength of this study as it allowed the identification of 2 important groups of cases. Firstly, there were some cases that had not been notified by the participating members of the specialist clinical networks. Secondly, it identified another group of infants who had a karyotype performed as part of routine evaluation because of some concern regarding sex development but in whom expert input from the specialist clinical networks was not sought during the neonatal period. Inclusion of this group for calculation of the birth prevalence of atypical genitalia where there is sufficient concern to perform a karyotype within the neonatal period provided a figure of $1: 1,881$, which is similar to that reported in a previous retrospective study [Rodie et al., 2011].

Despite this level of frequency of concerns regarding sex development at birth, the birth prevalence of delayed sex assignment defined as sex assignment at any time point beyond birth was only reported to occur in 1:11,097 term newborns, and there were no cases where sex was assigned after 2 weeks of age. In addition, there was only 1 case of sex reassignment within the first 3 months of life. Thus, over the study period, a delay in assigning sex beyond birth was an extremely rare occurrence, and although this was more likely in those infants who were XY and had less virilised genitalia, this was not always the case. The general recommendation is to delay sex assignment until completion of a thorough expert evaluation [Ahmed et al., 2016]. Given that an urgent query for X and $\mathrm{Y}$ specific probes may take 2 working days, the current study of contemporary practice in Scotland suggests that sex assignment as reported by the clinical staff is performed at a very early stage in cases of atypical genitalia and often well before seeking expert opinion or undertaking specialist investigations. Recent studies of temporal trends in sex assignment in DSD suggest that affected infants with an XY karyotype are more likely to be raised as male, irrespective of the extent of virilisation of the external genitalia [Kolesinska et al., 2018], and it is possible that this shift in practice may be another explanation for the relatively short time taken to assign sex in the current study. Given that parents themselves and the health care staff who were present at birth were not interviewed directly, it is unclear whether there was any uncertainty amongst parents regarding sex assignment in those cases who were reported by health care staff to have been assigned sex at birth. Interviews with parents with newborns with a wide range of genital atypicality do have concerns regarding long-term outcomes related to gender [Duiguid et al., 2007; Timmermans et al., 2019]. As this is the first systematic study of age at sex assignment, the reported data represent a new clinical benchmark that needs further study and comparison to practice elsewhere.

In the current study, the assessment of the involvement of the DSD multidisciplinary team in the care of newborn infants with DSD was compared against recent guidance for the UK [Ahmed et al., 2016]. Almost all infants that satisfied the reporting criteria were seen by a neonatalogist or a paediatrician and the majority by a pediatric endocrinologist or a pediatric surgeon/urologist within the first 3 months of life. However, only a small proportion were seen by a psychologist, and this was not dependent on whether there was any reported delay in sex assignment. Previous studies suggest that the appearance of the external genitalia may not be a good indicator of the extent of distress experienced by parents [Duiguid et al., 2007], and the reported practice in the current study seems to correspond to this observation. However, even then, psychological support had not been provided in the first 3 months in over $78 \%$ of cases where sex assignment was reported to have been delayed. It was reassuring that there was some evidence of targeting restricted psychology resources to those cases that were severely undermasculinised and had delayed sex assignment. Contemporary surveys of clinical psychology support that may be available for parents of these newborns report that many expert centres have ready availability of such professionals at an early stage following neonatal presentation [Pasterski et al., 2010; Kyriakou et al., 2016; Dessens et al., 2017; Rolston et al., 2017]. However, the current study is the first to provide objective data that quantifies the proportion of cases that were actually provided psychological support.

Along with pelvic imaging, more than half of the patients had biochemical investigations including urea and electrolytes, testosterone, anti-Müllerian hormone $(\mathrm{AMH})$, and androstenedione within the first few days of life. Other studies have reported similar findings, with testosterone being the most frequently performed biochemical investigation in this cohort of infants [Kyriakou
Rodie et al. 
et al., 2016]. On the other hand, hCG and ACTH stimulation tests are less frequently carried out, with these 'second tier' investigations only being performed if concerns have been raised from the initial workup [Kyriakou et al., 2016] in accordance with current UK guidance on the initial management of infants with atypical genitalia [Ahmed et al., 2016]. In comparison to the worldwide practice at expert centres [Kyriakou et al., 2016], the current data suggests that $\mathrm{AMH}$ and urinary steroid analysis are assessed more often, whilst the hCG stimulation test is performed less often within the first 3 months of life.

The data reported in this study are also valuable as, for the first time, they provide information on the birth prevalence of rare conditions associated with a DSD that may present in a term neonate with atypical genitalia. Within the broad category of conditions that are associated with a DSD and present with atypical genitalia in the XX newborn, CAH may be the most common single gene disorder [Khalid et al., 2012]. In the current study, it was interesting to note that over the 7.5-year period of the study, a total of 9 term infants with atypical genitalia were diagnosed with $\mathrm{CAH}$ within the neonatal period. Based on the total study term birth rate of 321,811 infants, this suggests a birth prevalence of $\mathrm{CAH}$ cases presenting in the neonatal period at 1:35,757, approximately half of what has been previously reported through a neonatal screening study performed in the same geographical region [Wallace et al., 1986] as well as more widely in the UK [Khalid et al., 2012]. This is likely to reflect the fact that boys with the common forms of CAH do not present with atypical genitalia in the neonatal period. The study also identified 3 infants with CAIS, providing an occurrence of this form of presentation of $1: 107,270$. Taking into account that some cases of CAIS may present in adulthood, these data are similar to that reported in a population-based study [Berglund et al., 2016].

\section{Conclusion}

This novel study that has prospectively and systematically collected data over a period of 7.5 years has found that although atypical genitalia that raise some concerns and require investigations in the neonatal period may occur in 1:1,881 term births, specialist input is sought in $1: 3,318$ term births, and delayed sex assignment is reported to occur in 1:11,097 term births. The study provides several indicators that can be used as clinical benchmarks for comparing and improving the delivery of care in centres that manage these complex conditions.

\section{Acknowledgements}

We would like to thank staff in the Scottish DSD Network, Scottish Paediatric Endocrine Group and the regional genetics laboratories including L. Bath, P. Batstone, S. Cascio, V. Cerqueira, J. Colgan, N. Conway, C. Driver, C. Durajczyk, D. Elleri, G. Farmer, M. Flett, S. Ghayyda, S. Imrie, E. Levy, K. Love, A. Mayo, A. Mason, B. McLennan, H. Miles, S. O’Toole, N. Pratt, G. Shaikh, D. Shanks, G. Stewart, A. Tasker, A. Webb. Dr Ali is supported by the Gardiner Lectureship at the University of Glasgow.

\section{Statement of Ethics}

The study was approved by the National Research Ethics Service in Scotland and the Caldicott Guardian as a health care evaluation survey.

\section{Conflict of Interest}

The authors have no conflicts of interest to disclose.

\section{Funding Source}

No funding was secured for this study.

\section{Author Contributions}

Professor Ahmed conceptualized and designed the study, coordinated and supervised data collection, and critically reviewed the manuscript for important intellectual content.

Dr Rodie and Dr Ali collected data, carried out the analyses, drafted the initial manuscript, and reviewed and revised the manuscript. Drs Jayasena, Alenazi, Cox, Cassim, Henderson, and McGowan and Mr McMillan collected data, carried out the initial analyses, and reviewed and revised the manuscript. All authors approved the final manuscript as submitted and agree to be accountable for all aspects of the work.

References

Acherman JC. Hughes IA. Chapter 23 Pediatric Disorders of Sex Development. In: Melmed S, Polonsky KS, Larsen PR, Kronenberg HM, editors. Williams Textbook of Endocrinology. Section VI B: Childhood. Philadelphia: Elsevier; 2017.

Ahmed SF, Khwaja O, Hughes IA. The role of a clinical score in the assessment of ambiguous genitalia. BJU Int. 2000;85(1):120-4.

Ahmed SF, Dobbie R, Finlayson AR, Gilbert J, Youngson G, Chalmers J, et al. Prevalence of hypospadias and other genital anomalies among singleton births, 1988-1997, in Scotland. Arch Dis Child Fetal Neonatal Ed. 2004; 89(2):F149-51. 
Ahmed SF, Achermann JC, Arlt W, Balen A, Conway G, Edwards Z, et al. Society for Endocrinology UK guidance on the initial evaluation of an infant or an adolescent with a suspected disorder of sex development (Revised 2015). Clin Endocrinol (Oxf). 2016;84(5):771-88.

Aydin BK, Saka N, Bas F, Bas EK, Coban A, Yildirim S, et al. Frequency of ambiguous genitalia in 14,177 newborns in Turkey. J Endocr Soc. 2019;3(6):1185-95.

Berglund A, Johannsen TH, Stochholm K, Viuff $\mathrm{MH}$, Fedder J, Main KM, et al. Incidence, prevalence, diagnostic delay, and clinical presentation of female 46,XY disorders of sex development. J Clin Endocrinol Metab. 2016; 101(12):4532-40

Dessens A, Guaragna-Filho G, Kyriakou A, Bryce J, Sanders C, Nordenskjöld A, et al. Understanding the needs of professionals who provide psychosocial care for children and adults with disorders of sex development. BMJ Paediatr Open. 2017;1(1):e000132.

Duiguid A, Morrison S, Robertson A, Chalmers J, Youngson G, Ahmed SF, Scottish Genital Anomaly Network. The psychological impact of genital anomalies on the parents of affected children. Acta Paediatr. 2007;96(3):348-52.
Hughes IA, Houk C, Ahmed SF, Lee PA; LWPES Consensus Group; ESPE Consensus Group. Consensus statement on management of intersex disorders. Arch Dis Child. 2006;91(7): 554-63.

Khalid JM, Oerton JM, Dezateux C, Hindmarsh PC, Kelnar CJ, Knowles RL. Incidence and clinical features of congenital adrenal hyperplasia in Great Britain. Arch Dis Child. 2012; 97(2):101-6.

Kolesinska Z, Acierno J, Ahmed SF, Xu C, Kapczul K, Skorczyk-Werner A, et al. Integrating clinical and genetic approaches in the diagnosis of 46,XY disorder of sex development. Endocr Connect. 2018; 7(12):1480-90.

Kyriakou A, Dessens A, Bryce J, Iotova V, Juul A, Krawczynski M, et al. Current models of care for disorders of sex development - results from an international survey of specialist centres. Orphanet J Rare Dis. 2016;11(1):155.

Pasterski V, Prentice P, Hughes IA. Consequences of the Chicago consensus on disorders of sex development (DSD): current practices in Europe. Arch Dis Child. 2010;95(8):618-23.

Rodie M, McGowan R, Mayo A, Midgley P, Driver CP, Kinney M, et al. Factors that influence the decision to perform a karyotype in suspected disorders of sex development: lessons from the Scottish genital anomaly network register. Sex Dev. 2011;5(3):103-8.
Rolston AM, Gardner M, van Leeuwen K, Mohnach L, Keegan C, Delot E, et al. Disorders of sex development (DSD): Clinical service delivery in the United States. Am J Med Genet C Semin Med Genet. 2017;175(2):268-78.

Sandberg DE, Gardner M, Cohen-Kettenis PT. Psychological aspects of the treatment of patients with disorders of sex development. Semin Reprod Med. 2012;30(5):443-52.

Thyen U, Lanz K, Holterhus PM, Hiort O. Epidemiology and initial management of ambiguous genitalia at birth in Germany. Horm Res. 2006;66:195-203.

Timmermans S, Yang A, Gardner M, Keegan CE, Yashar BM, Fechner PY, et al. Gender destinies: assigning gender in Disorders of Sex Development-Intersex clinics. Sociol Health Illn. 2019;41(8):1520-34.

Wallace AM, Beastall GH, Cook B, Currie AJ, Ross AM, Kennedy R, et al. Neonatal screening for congenital adrenal hyperplasia: a programme based on a novel direct radioimmunoassay for 17-hydroxyprogesterone in blood spots. J Endocrinol. 1986;108(2):299-308. 